\title{
Homotopic Labeling of Elements in a Tetrahedral Mesh for the Head Modeling
}

\author{
Jasmine Burguet and Isabelle Bloch \\ École Nationale Supérieure des Télécommunications \\ Département TSI - CNRS UMR 5141 LTCI \\ 46 rue Barrault \\ 75634 Paris Cedex 13, France \\ \{jasmine. burguet, isabelle.bloch\}@enst.fr
}

\begin{abstract}
In this paper we propose a method to obtain a tetrahedral model of the human head by labeling elements of a tetrahedral mesh. To work with meshes as regular as possible, we use the notion of Almost Regular Tesselation (ART) providing tetrahedral meshes with good quality elements. The proposed labeling method uses segmented M.R.I. containing main tissues of the head as input. The labeling is done under topological constraints in order to preserve topological arrangement of the head tissues. This process uses a notion of simple tetrahedra.
\end{abstract}

\section{Introduction}

In applications based on Electroencephalography (E.E.G.) and Magnetoencephalography (M.E.G.) data, electromagnetic field propagation is numerically calculated, for example using finite element methods. This computation is based on a meshed model of the head tissues generally obtained thanks to Magnetic Resonance Imaging (M.R.I.), in order to get realistic models. The purpose of this paper is to propose a method allowing us to build such head models, we focus particularly on the mesh generation description and the labeling of the tetrahedra according to the anatomical tissues. One main constraint of our method is the preservation of the topological arrangement of the head structures. Indeed, the literature mainly concerns the mesh construction, refinement or adaptation, but very few references deal with topological aspects, which is however an important criterion. The input image is a segmented M.R.I. containing the repartition of the main tissues of the head (for instance the brain, skull or scalp). Then we can assign the corresponding property (for example electrical conductivity) to each segmented tissue. The segmentation step is outside the scope of this paper but more details can be found in $[1,2]$. Next we build the tetrahedral mesh. The quality of the tetrahedra influences the accuracy of the numerical computations. So bad shaped tetrahedra (sliver, cap or needle for instance) must be avoided. Ideally a Finite Element (F.E.) mesh should be entirely composed of regular tetrahedra, but unfortunately we cannot tessellate $\mathbb{R}^{3}$ only with equilateral tetrahedra. The common approaches to construct the meshes use a polyhedral representation of the boundary of the object to tessellate. The principle is the 
introduction of points in the interior of the object, and then a Delaunay tesselation is applied to generate the finite elements [3-6]. One drawback of these methods is the necessity of post-treatment to remove bad quality tetrahedra. The complexity of the head structures (see the brain for instance) and the use of M.R.I. (and so discrete surfaces instead of polyhedral ones) as input make the application of these methods very difficult. These are the reasons why we use for our purpose the Almost Regular Tesselation (ART) introduced by J. Pescatore which produces a tesselation made of good quality tetrahedra [2, 7]. Finally, after the tetrahedral mesh generation, we must label the tetrahedra according to their membership to the different segmented head tissues. It is a well known fact that a topology of interwoven spheres [8,9] is a good approximation of the topology of head tissues. Therefore it is important to use a homotopic labeling method, i.e. a method under topological constraints, in order to prevent a bad configuration like a contact between the scalp and the brain. J. Pescatore proposed one based on the use of simple tetrahedra [2]. Using his algorithm, the topology of full sphere was guaranteed for the brain and the whole head. However a topology of empty sphere was not guaranteed for the skull or the scalp for instance. Up to now, no other methods takes care about the tetrahedral meshes topology. However, even if the segmented image satisfies the wanted topology, there is absolutely no guarantee that it is the case for the labeled mesh if we directly label the tetrahedra according to the segmentation, in particular if some segmented structures are very thin. Moreover, the numerical methods used to compute the electromagnetic field propagation are very sensitive to these bad configurations. So we propose a new labeling procedure that respects a spherical topology for all structures, that is the main contribution of our work.

The paper is organized as follows: Section 2 introduces the ART, presents the images we use as input and presents some initializations. Then Section 3 exposes the different topological tools we need for our method, like the tetrahedra simplicity or the notions of thinning/thickening. The labeling process is described in Section 4 and some results are proposed. Finally we discuss the obtained results and propose some perspectives to this work in Section 5.

\section{Tetrahedral Mesh and Initializations from Segmented M.R.I.}

Here we present the construction of a tetrahedral mesh called Almost Regular Tesselation (ART) (see [2,7] for more details). A tetrahedral tesselation of $\mathbb{R}^{3}$ is Almost Regular if it is possible to tessellate $\mathbb{R}^{3}$ with tetrahedra such that each tetrahedron has a fixed connectivity. The principle of the ART construction is based on the following notion: a tetrahedron $T$ is said to be subdivision invariant (SI) if we can divide $T$ into 8 tetrahedra which are congruent to $T$ (scaled by a factor $1 / 2$ ) by halving the edges of $T$. Note that the regular tetrahedron is not subdivision invariant. Figure 1 shows an example of SI tetrahedron. Using such a tetrahedron we can generate an ART having the following properties: 


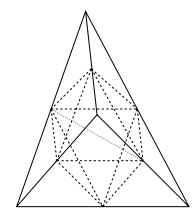

Fig. 1. Example of SI tetrahedron and its subdivision into 8 tetrahedra.
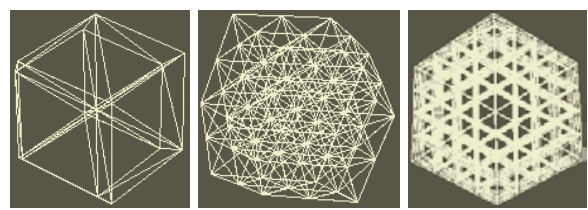

Fig. 2. ART construction with from left to right: $n=0, n=1$ and $n=2$.

- it is structured: a tetrahedron has 4 neighbors per face, 18 neighbors per edge, 70 neighbors per vertex; a vertex is shared by 24 tetrahedra;

- it is conform: the intersection of two tetrahedra is either $\emptyset$, a face, an edge or a vertex.

To obtain a tesselation as regular as possible, we must use a SI tetrahedron having a good quality. There are several ways to evaluate this quality [10,11], such as: $Q_{T_{\alpha}}=\alpha \frac{h_{\max }}{\rho_{T}}$ where $h_{\max }$ is the largest edge of $T, \rho_{T}$ the in-radius of $T$ and $\alpha$ a regulation coefficient $\left(\alpha=\sqrt{6} / 12\right.$ for $Q_{T_{\alpha}}=1$ if $T$ is the regular tetrahedron). Since $Q_{T_{\alpha}}$ varies from 1 to $\infty$, we consider $Q_{T_{\alpha}}^{-1}$ that varies from 0 to 1 . As shown in [7], we choose the following SI tetrahedron since it has an optimal quality (i.e. the closest to 1 ):

$$
T^{*}=\left\{\left(\begin{array}{l}
0 \\
0 \\
0
\end{array}\right),\left(\begin{array}{l}
1 \\
0 \\
0
\end{array}\right),\left(\begin{array}{c}
\frac{1}{3} \\
\frac{2 \sqrt{2}}{3} \\
0
\end{array}\right),\left(\begin{array}{c}
\frac{2}{3} \\
\frac{\sqrt{2}}{3} \\
\frac{2}{3}
\end{array}\right)\right\}, Q_{T_{\alpha}^{*}}^{-1}=0.866 .
$$

For our purpose, we build the ART initialization as the polyhedron $P$ composed of 24 tetrahedra $T^{*}$ sharing a common vertex. This vertex is chosen as the center of the segmented head $S(I)$, and we use a scale factor $S$ to enlarge $P$ until it contains entirely $S(I)$. Next, each tetrahedron is recursively subdivided $n$ times until the desired precision is reached (see Figure 2). The final number of tetrahedra of the ART is $N_{n}=24 * 8^{n}\left(N_{4}=98304\right.$ and $\left.N_{5}=786432\right)$. Moreover each tetrahedron of the ART has the same quality as $T^{*}$.

Our input images are segmented from M.R.I of human heads. Let $I$ be a M.R.I. We suppose that the segmentation $S(I)$ of $I$ contains $n_{t}$ tissues of interest. In this paper we use a segmentation program developed in [1] producing segmented images under constraints to preserve the topology (see Figure 3). We associate an index $j$ to each tissue, $1 \leq j \leq n_{t}$, such that the tissue $j$ is bounded by the tissue $j+1$, and $j=1$ corresponds to the brain. The index $j=0$ corresponds to the background of the image. Figure 4 presents the general scheme of the head and an example for $n_{t}=3$.

Before the labeling, we associate to each tetrahedron $t$ a vector $T a b(t)$ of size $n_{t}+1$ containing the percentages of each component $j$ (background or tissue) in $t$. The vectors are computed thanks to $S(I)$. The value $T a b(t)[0]$ corresponds to the percentage of background in $t, \operatorname{Tab}(t)[1]$ to the percentage of brain and so on. For instance, if $n_{t}=3$ and $\operatorname{Tab}(t)=[0.11,0.62,0.27,0.0]$, then $t$ contains 

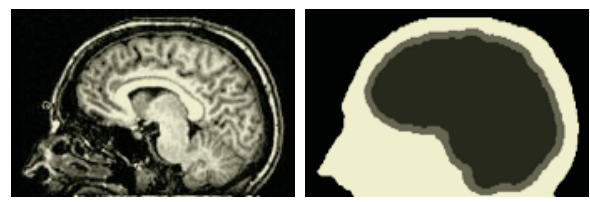

Fig. 3. M.R.I $I$ (left) and segmentation $S(I)$ of $I$ into Brain, Skull and Scalp.
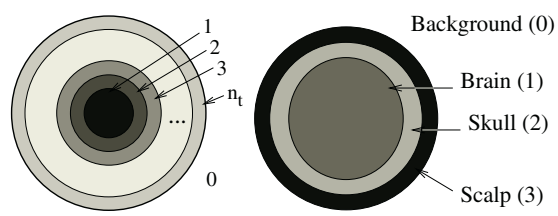

Fig. 4. General scheme of the head for $n_{t}$ tissues (left) and for $n_{t}=3$ (right).

$11 \%$ of background, $62 \%$ of brain, $27 \%$ of skull and $0 \%$ of Scalp. We define $P_{\text {Head }}(t)=\sum_{i=1}^{n_{t}} \operatorname{Tab}(t)[i]=1-\operatorname{Tab}(t)[0]$.

\section{Topological Tools}

\subsection{Definitions}

Our labeling method uses topological tools defined in [2,7]. First let us introduce some basic concepts. We suppose that vertices, edges, faces and tetrahedra contain their boundaries and they are also called $0-, 1-, 2-$ and 3 -simplexes, respectively. Let $O$ be a set of tetrahedra and $k$ an integer such that $k<3$.

A $k$-simplex $s$ is said to be shared if there exist two tetrahedra $t_{1}, t_{2} \in O$ such that $s \in t_{1} \cap t_{2}$. Otherwise, $s$ is called bare. Let $t$ be a tetrahedron. The neighborhood $V(t)$ of $t$ is the union of all tetrahedra $t_{i}$ such that $t_{i} \cap t \neq \emptyset$. We denote by $B d(t)$ the boundary of $t$ defined as the union of the whole faces of $t$. The attachment set of $t B d_{s}(t)$ is the union of all the shared $k$-simplexes of $t$, and the bare set of $t$ is $B d_{b}(t)=B d(t) \backslash B d_{s}(t)$. Two simplexes $s_{1}$ and $s_{2}$ are said to be adjacent if and only if $s_{1} \cap s_{2} \neq \emptyset$. A set of simplexes $s_{1}, s_{2}, \ldots, s_{k}$ in which each $s_{i}$ is adjacent to $s_{i+1}, 1 \leq i<k$, is called a path of simplexes from $s_{1}$ to $s_{k}$. Then the set of tetrahedra $O$ is said to be connected if for any pair of tetrahedra $t, t^{\prime} \in O$, there exists a path of simplexes $s_{1}, s_{2}, \ldots, s_{k}$ such that $s_{1}=t, s_{k}=t^{\prime}$ and, for $i=1, \ldots, k, s_{i} \in O$. A maximal connected subset of $O$ is called connected component of $O$.

We suppose that $O$ is connected. Following Kong [12] we define the simplicity of a tetrahedron as follows:

Definition 1. Let $t$ be a tetrahedron and $O$ a connected set of tetrahedra. Then $t$ is said to be simple if there exits a homotopy equivalence from $O$ to $O \cup t$ or from $O$ to $O \backslash\{t\}$.

There exists a local characterization of the simplicity of a tetrahedron:

Theorem 1 (from [2]). The tetrahedron $t$ is simple in $O$ if and only if $B d_{s}(t)$ and $B d_{b}(t)$ are not empty and are connected.

In Figure 5, let us consider the tetrahedra $t_{1}$ and $t_{2}$ and their attachment sets. In (a), the tetrahedron $t_{1}$ is simple in $O_{1}$ since $B d_{s}\left(t_{1}\right)$ and $B d_{b}\left(t_{1}\right)$ are not empty and are connected. In (b), $t_{2}$ is not simple in $O_{2}$ because $B d_{s}\left(t_{2}\right)$ is not connected. 


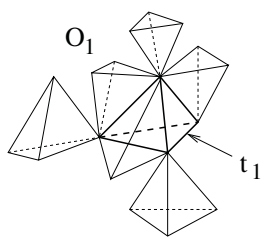

(a)

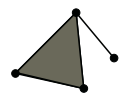

$\mathrm{Bd}_{\mathrm{s}}\left(\mathrm{t}_{1}\right)$

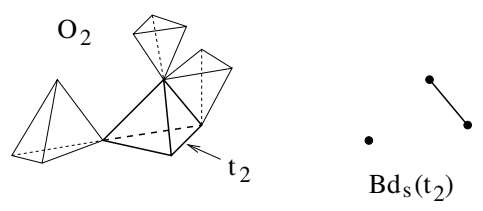

(b)

Fig. 5. Two tetrahedra $t_{1}$ and $t_{2}$ (left) and their attachment sets (right).
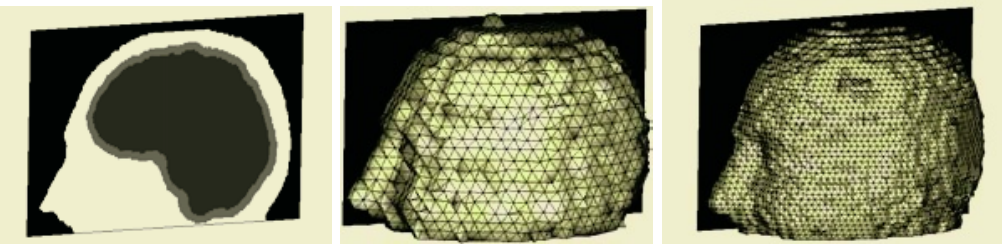

Fig. 6. Result of the head construction for $n=4$ (middle) and $n=5$ (right).

\subsection{Homotopic Thinning / Thickening}

Based on the use of simple tetrahedra, we define the following notions:

Definition 2. We call homotopic thinning (resp. homotopic thickening) of a connected set of tetrahedra $O$ the sequential deletion (resp. addition) of simple tetrahedra in $O$. A set of tetrahedra $S^{\prime}$ obtained by a homotopic thinning or homotopic thickening of a set $S$ is said to be homotopic to $S$.

One first application of these notions is the constitution of the set containing the tetrahedra of the head among all the tetrahedra of the ART. First we choose a tetrahedron $t$ which belongs to the head. For example, if $n_{t}=3$, we choose a $t$ such that $\operatorname{Tab}(t)=[0.0,1.0,0.0,0.0]$. Then we perform a homotopic thickening of the set $H=\{t\}$ : we add to $H$ simple tetrahedra $x$ such that $P_{\text {Head }}(x)>$ $\mu_{\text {Head }}$ where $\mu_{\text {Head }} \geq 0$ is a parameter of the method. For instance, if $\operatorname{Tab}(x)=$ $[0.71,0.18,0.11,0.00]$ and $\mu_{\text {Head }}=0.10$, then $P_{\text {Head }}(x)=0.29 \geq \mu_{\text {Head }}$, so $x$ can be added to $H$ during the thickening if $x$ is also simple. The resulting set corresponds to the head and is homotopic to a single tetrahedron, i.e. to a full sphere. We can see in Figure 6 the result of this labeling for $n=4$ and $n=5$, with $S=160$ and $\mu_{\text {Head }}=0$.

One new tool we propose to label the different tissues of the head is the original notion of interior thinning. First, a tetrahedron $t$ is said to be interior to a set of tetrahedra $O$ if $t \in O$ and if $V(t) \subset O$. Then we say that a tetrahedron is removable if it is simple and if it also satisfies another condition (for instance $\{\operatorname{Tab}(t)[2](x)<0.5\})$. An interior thinning of a set $H$ begins with the choice of an interior tetrahedron $t$ in $H$. Then, we apply a homotopic thinning to the set $H \backslash t$ (which is homotopic to an empty sphere) starting from $t$ and deleting removable tetrahedra until stability. The corresponding pseudocode is: 
- list_of tetrahedra $l_{1} \leftarrow$ neighbors by face of $t$; // border of the thinning

- list_of - tetrahedra $l_{2} \leftarrow$ NULL;

$/ /$ next border of the thinning

- tetrahedron $t \leftarrow$ interior_tetrahedron;

Iterate until stability

for each tetrahedron $x$ in $l_{1}$

if $x$ is removable then delete $x$ from $H$ and update $l_{2}$;

else put $x$ into $l_{2}$;

$l_{1} \leftarrow l_{2} ;$

$l_{2} \leftarrow$ NULL;

Since we delete only simple tetrahedra during this thinning, the set of deleted tetrahedra is homotopic to a full sphere whereas the set of remaining tetrahedra $R$ is homotopic to an empty sphere. An example of the result of an interior thinning applied to the head $H$ is shown in Figure 7 (a tetrahedron was removable if it was only simple, without additional condition).
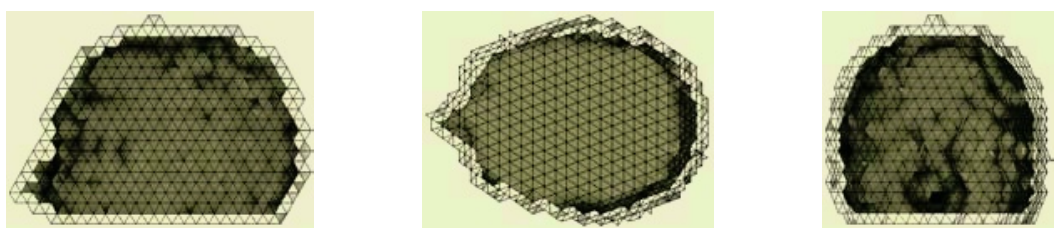

Fig. 7. Result of an interior thinning of the head by deleting simple tetrahedra (light gray set).

\section{Homotopic Tissues Labeling}

We now propose a new complete labeling scheme. We label the tissues from the most exterior to the most interior one, thus from $j=n_{t}$ to $j=1$. Let $O \subset H$ be the set of tetrahedra which are not yet labeled (initially $O=H$ and $j=n_{t}$ ).

First we choose a tetrahedron $t$ interior to $O$ such that $\mathrm{Tab}(\mathrm{t})[1]=1(t$ contains only brain). Then we iterate the following process while $j>1$ :

1. We apply an interior thinning to the set $O$, a tetrahedron $x$ being removable if it satisfies the two following properties:

$-x$ is simple,

- there exists a tissue $i$ such that $0<i \leq n_{t}, i \neq j, \operatorname{Tab}(t)[i]>\operatorname{Tab}(t)[j]$.

The tetrahedra of the remaining set $R$ are labeled as $j$.

2. The set $O$ becomes $O \backslash R, j$ becomes $j-1$, and we eventually update $t$.

Let us consider Figure 8. In (a) the set $O$ and the interior tetrahedron $t$ are shown. We see in (b) the result of the labeling for the tissue $j$. Next, in Figure 8 (c) and (d) we can see the labeling of tissue $j-1$ obtained with the updated set $O$. After the labeling for all $j>1$, we label the final set $O \backslash R$ (i.e. the removed tetrahedra during the labeling of tissue 2) with $j=1$ (i.e. the brain). 


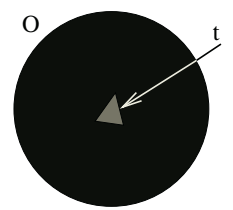

(a)

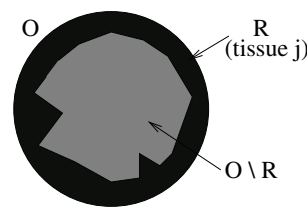

(b)

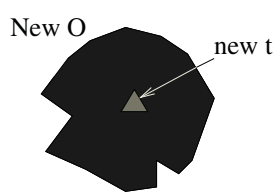

(c)

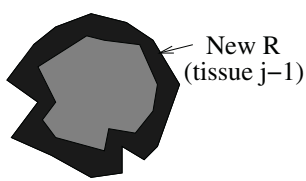

(d)

Fig. 8. Labeling of tissues such that $j>2$.

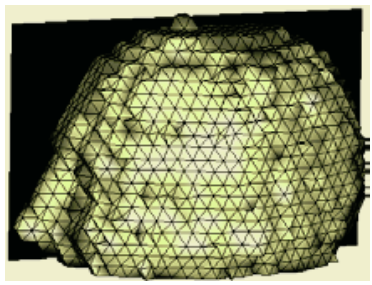

13187 tetrahedra

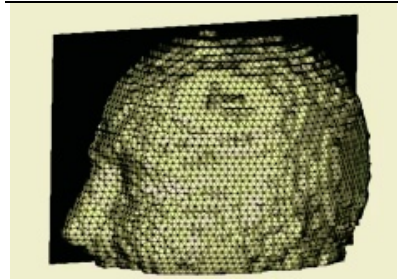

94893 tetrahedra

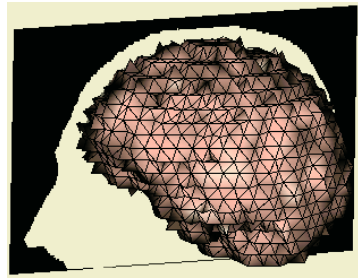

5647 tetrahedra

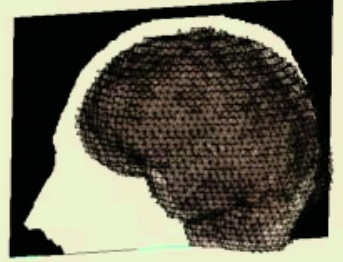

26453 tetrahedra

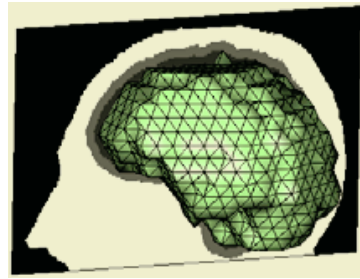

8210 tetrahedra

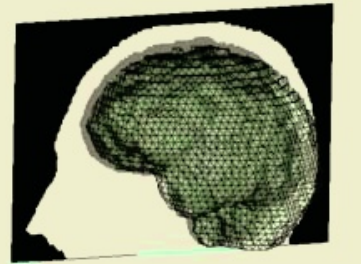

82399 tetrahedra

Fig. 9. Result of the head labeling for the scalp, skull and brain (from left to right), for $n=4$ (top) and $n=5$ (bottom), with $S=160$.

After the labeling for all $j>1$, we label the final set $O \backslash R$ (i.e. the removed tetrahedra during the labeling of tissue 2) with $j=1$ (i.e. the brain).

In Figure 9, the results (exterior surfaces) of the topological labeling are shown for the scalp, skull and brain respectively (the number of tetrahedra for each tissue is also given).

\section{Conclusion and Perspectives}

We proposed in this paper a way to build a tetrahedral model of the head. First we constructed an ART mesh to tessellate a volume containing the head. Then, using a segmented M.R.I. of the head, we defined a homotopic process to label tetrahedra of the ART in order to get a tetrahedral head. Our method guarantees that the resulting head has a topology of interwoven spheres. Examples shown in the paper are obtained for $n_{t}=3$ tissues, but our method is adapted to any value of $n_{t}$, as soon as these tissues have a spherical topology (we can moreover consider the Cerebro-Spinal Fluid for example). But the use of a segmentation having a non-adequate topology (for example segmentation with isolated components or 
including the jaw in the skull) can produce results with bad a geometrical quality. One perspective of this work is to increase the robustness of our method for any type of segmented head, by releasing topological constraints during the labeling.

In several applications like the study of the impact of the exposure to electromagnetic waves from mobile phones on human health, we may want to focus our attention on precise places, like the ear. A multi-scale approach could be of high interest to model important localizations with a higher degree of accuracy.

\section{References}

1. Dokladal, P., Bloch, I., Couprie, M., Ruijters, D., Urtasun, R., Garnero, L.: Topologically Controlled Segmentation of 3D Magnetic Resonance Images of the Head by using Morphological Operators. Pattern Recognition 36 (2003) 2463-2478

2. Pescatore, J.: Maillages Homotopiques Tétraédriques des Tissus de la Tête pour le Calcul du Problème Direct en Électro/Magneto-Encéphalographie. PhD thesis, École Nationale Supérieure des Télécommunications (2001)

3. George, J.: Computer Implementation of the Finite Element Method. PhD thesis, Dept. of Computer Science Stanford University (1971)

4. George, P., Séveno, E.: The Advancing Front Mesh Method Revisited. Internation Journal in Numerical Methods in Engineering 37 (1994) 3605-3619

5. Peraire, J., Morgan, K.: Unstructured Mesh Generation Including Directional Refinement for Aerodynamics Flow Simulation. Finite Elements in Analysis and Design 25 (1997) 343-355

6. Bowyer, A.: Computing Dirichlet Tesselations. The Computer Journal 24 (1981) 162-167

7. Pescatore, J., Bloch, I., Baillet, S., Garnero, L.: FEM Tetrahedral Mesh of Head Tissues from MRI under Geometric and Topological Constraints for Applications in EEG and MEG. In: Human Brain Mapping HBM 2001, Brighton, UK (2001) 218

8. Munck, J.: The Potential Distribution in a Layered Anisotropic Spheroidal Volume Conductor. Journal of applied Physics 64 (1988) 464-470

9. Sarvas, J.: Basic Mathematical and Electromagnetic Concepts of the Biomagnetics Inverse Problem. Physics in Medical Biology 32 (1987) 11-22

10. Parthasarathy, V., Graichen, C., Hathaway, A.: A Comparison of tetrahedron Quality Measures. Finite Elements in Analysis and Design 15 (1993) 255-261

11. Martinez, E., Garnero, L., Bloch, I.: Refined Adaptive Meshes for the Localization of Cortical Activity. Technical Report 2003D001, Ecole Nationale Supérieure des Télécommunications (2003)

12. Kong, T.Y.: On Topology Preservation in 2D and 3d Thinning. Internation Journal of Patterne Recognition and Artificial Intelligence 9 (1995) 813-844 